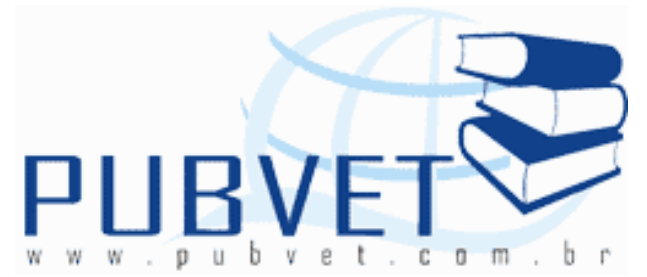

PUBVET, Publicações em Medicina Veterinária e Zootecnia.

\title{
Parâmetros genéticos para características de produção em linhagem macho de matrizes de frango de corte: Herdabilidades e correlações
}

\section{Raquel Grden Szinvelski ${ }^{1}$ Stela Adami Vayego², Nelson José Laurino Dionello3, Elsio Antonio Pereira de Figueiredo ${ }^{4}$, Heloísa Cristina Clemente ${ }^{5}$, Aline Oliveira Stocco $^{6}$}

${ }^{1}$ Graduanda de Medicina Veterinária/UFPR - Bolsista de Iniciação

Científica/Fundação Araucária

2Professora do DEST/UFPR;

${ }^{3}$ Professor do DZ/FAEM/UFPEL;

${ }^{4}$ Pesquisador da EMBRAPA Suínos e Aves;

${ }^{5}$ Graduanda de Zootecnia/UFPR;

${ }^{6}$ Graduanda de Zootecnia/UFPR.

\section{Resumo}

As estimativas de parâmetros genéticos para algumas características de produção em linhagem de matrizes de frango de corte foram obtidas usando dados fornecidos pela EMBRAPA Suínos e Aves, Concórdia/SC. As características avaliadas foram: peso corporal aos 42 dias de idade (P42), comprimento de peito (CPeito), largura maior (Larg1) e largura menor (Larg2) do peito aos 42 dias, fertilidade (Fert), eclodibilidade (Eclod), nascimento (Nasc), peso aos 49 dias (P49), ganho de peso (GP4349) e conversão alimentar (CA4349) entre os dias 43 e 49. Os valores das estimativas das 

macho de matrizes de frango de corte: Herdabilidades e correlações. PUBVET, Londrina, V. 5, N. 35, Ed. 182, Art. 1230, 2011.

herdabilidades foram consideradas de baixas a moderadas, variando entre 0,0103 a 0,5352. As herdabilidades estimadas para as características de fertilidade, ganho de peso, conversão alimentar, nascimento e peso aos 49 dias de idade foram: 0,0103 $\pm 0,190,0,0757 \pm 0,044,0,0912 \pm 0,043$, $0,0930 \pm 0,218$, 0,1472 $\pm 0,053$, respectivamente; e 0,2794 $\pm 0,057$, $0,2943 \pm 0,061,0,3484 \pm 0,06$, para largura menor, largura maior e peso aos 42 dias de idade respectivamente. Estimativas altas de herdabilidade foram encontradas nas características eclodibilidade e comprimento de peito, com os valores de 0,5053 $\pm 0,334$ e 0,5352 $\pm 0,066$, respectivamente.

Palavras-chave: matrizes de frango de corte, parâmetros genéticos, DFREML

\section{Genetic parameters for production characteristics in male lineage of arrays of broilers: heritabilities and correlations}

\section{Abstract}

Estimates of genetic parameters for production traits in the lineage of arrays of broiler chickens were obtained from a database supplied by Embrapa Swine and Poultry, Concórdia/SC. The characteristics evaluated were: body weight at 42 days old (P42), breast length (CPeito), greater width (Larg1) and smaller width (Larg2) of the breast at 42 days, fertility (Fert), hatchability (Eclod), birth (Nasc), weight at 49 days (P49), gain weight (GP) and feed conversion (CA) between days 43 and 49 . The estimates of heritability were considered low to moderate, changing from 0.0103 to 0.5352 . The values with moderate to high heritability respond better to selection, while the low heritability respond to environmental variation. The values of heritability from fertility, weight gain, feed conversion, birth and weight at 49 days were: $0.0103 \pm$ $0.190,0.044 \pm 0.0757,0.0912 \pm 0.043,0.218 \pm 0.0930,0.1472 \pm 0.053$, respectively; and $0.2794 \pm 0.057,0.061 \pm 0.2943,0.3484 \pm 0.06$, in smaller width, greater width and weight at 42 days. High estimates of heritability were found in hatchability and length of breast, with the values of $0.5053 \pm 0.5352$ \pm 0.334 and 0.066 , respectively.

Keywords: arrays of broiler, genetic parameters, DFREML 

macho de matrizes de frango de corte: Herdabilidades e correlações. PUBVET, Londrina, V. 5, N. 35, Ed. 182, Art. 1230, 2011.

\section{INTRODUÇÃO}

A avicultura é uma atividade da zootécnica que apresentou os maiores índices de evolução nas últimas décadas. Nos anos compreendidos entre 1992 e 2001, a produção de carne de frango aumentou $128 \%$ e em $236 \%$ a exportação (BOARETTO, 2009). A avicultura de corte assegura ao Brasil posição de destaque no cenário mundial. A partir de 2004, o país passou a ser o maior exportador mundial de carne de frango, à frente dos Estados Unidos da América, bem como o terceiro maior produtor, à frente de 25 países da União Européia (ABEF, 2007; citado por NUNES, 2007). O Brasil é hoje um dos maiores produtores e exportadores de carne de frango do mundo, com cerca de 8,4 milhões de toneladas produzidas ficando atrás apenas dos Estados Unidos e China (BOARETTO, 2009). Esse desempenho é resultado de uma trajetória de incremento tecnológico e capacidade de coordenação entre os diferentes segmentos de produção que compõem o setor avícola.

A evolução no desempenho das linhagens de frango de corte tem sido acentuada nas últimas décadas, sendo ao redor de $85 \%$ a $90 \%$ dos ganhos em produtividade de origem genética (CHAMBERS, 1995). Em linhagens de corte, o melhoramento genético tem determinado ganhos anuais da ordem de 2,5; 0,25 e 0,15\%, respectivamente, para peso corporal, rendimentos de carcaça e de peito, além da redução, da ordem de 0,02 unidades, 0,1\% e 0,4 dias, respectivamente, na conversão alimentar, gordura abdominal e idade de abate (ANDRADE, 1990).

Novas técnicas de melhoramento genético têm surgido com o intuito de aumentar a produção e desenvolver ainda mais o setor avícola, levando em consideração sempre o objetivo do melhoramento genético é a melhoria continua das características de interesse, maximizando assim a produção de carne de alta qualidade com o mínimo custo, já que a atividade no setor tem evoluído muito nos últimos anos, e a preferência dos consumidores pela carne de frango ter aumentado significativamente (BOARETTO, 2009). 

macho de matrizes de frango de corte: Herdabilidades e correlações. PUBVET, Londrina, V. 5, N. 35, Ed. 182, Art. 1230, 2011.

Segundo CAMPOS \& PEREIRA (1999) a estimação das herdabilidades e das correlações genéticas, permitem estabelecer estratégias de melhoramento e o monitoramento da variabilidade genética dos indivíduos das linhagens utilizadas.

Portanto, este estudo teve como objetivo estimar os parâmetros genéticos (herdabilidades e correlações) e fenotípicos para algumas características de produção em linhagem paterna de matrizes de frango de corte.

\section{MATERIAL E MÉTODOS}

O estudo foi desenvolvido utilizando-se informações de parentesco de matrizes macho de frangos de corte, do Banco Ativo de Germoplasma para Aves da EMBRAPA Suínos e Aves em Concórdia/SC.

Foram avaliadas as características de peso corporal aos 42 dias de idade (P42), comprimento de peito (CPeito) aos 42 dias de idade, largura maior do peito (Larg1) aos 42 dias de idade, largura menor do peito (Larg2) aos 42 dias de idade, fertilidade (Fert), eclodibilidade (Eclod), nascimento (Nasc), peso aos 49 dias de idade (P49), ganho de peso (GP) e conversão alimentar (CA) compreendido entre os 43 e 49 dias de idade.

Após tratamento dos dados, aves com identificação confusa, sem nenhuma informação ou informações duplicadas foram identificadas e removidas do banco de dados.

O cálculo das médias, desvios-padrão, coeficientes de variação, valores mínimo e máximo de cada característica foram realizados através do programa de domínio público Bioestat 5.0.

Para a estimação das herdabilidades e correlações genéticas foi utilizado o programa de domínio público DFREML (Derivative-Free resticted Maximum Likelihood - versão 3.0), desenvolvido por MEYER (1988), que emprega o AMBLUP, através dos pacotes DFUNI, para análises univariada e DXMUX para análises bivariável. 

macho de matrizes de frango de corte: Herdabilidades e correlações. PUBVET, Londrina, V. 5, N. 35, Ed. 182, Art. 1230, 2011.

Utilizou-se o seguinte modelo estatístico, em sua forma matricial:

$$
\mathrm{y}=\mathrm{X} \underset{\sim}{\beta}+\mathrm{Z} \underset{\sim}{\mathrm{u}}+\underset{\sim}{\mathrm{e}}
$$

onde $\underset{\sim}{\mathrm{y}}$ : é o vetor (nqx1) de observações de q características medidas organizadas dentro cada animal, sendo n o número de animais em avaliação; $\underset{\sim}{\beta}$ : é o vetor $(p q \times 1)$ de efeitos fixos, com $p$ o número de efeitos fixos; $X$ : é a matriz (nqxpq) de incidência de p efeitos fixos; $\underset{\sim}{u}$ : é o vetor (nqx1) de efeitos aleatórios (efeitos genéticos aditivos dos animais); Z: é uma matriz diagonal (nqxnq) de incidência de efeitos aleatórios, constituída de "1" ou " 0 ", na diagonal principal, dependendo se a característica foi medida ou não, no animal e $\underset{\sim}{e}$ : é o vetor (nqx1) de variáveis aleatórias não observáveis, dito vetor de erros aleatórios.

As pressuposições da distribuição multivariada de $\underset{\sim}{\mathrm{y}}, \underset{\sim}{\mathrm{u}}$ e $\underset{\sim}{\mathrm{e}}$ são:

$$
\left[\begin{array}{c}
\mathrm{y} \\
\tilde{u} \\
\tilde{\mathrm{e}} \\
\sim
\end{array}\right] \sim \mathrm{N}\left\{\left[\begin{array}{c}
\mathrm{X} \beta \\
\tilde{0} \\
\tilde{\tilde{0}} \\
\sim
\end{array}\right] ;\left[\begin{array}{ccc}
Z G Z^{\prime}+\mathrm{R} & \mathrm{ZG} & \mathrm{R} \\
\mathrm{GZ} & \mathrm{G} & \phi \\
\mathrm{R} & \phi & \mathrm{R}
\end{array}\right]\right\} \text {. }
$$

Estando as medidas ordenadas dentro de cada animal no vetor $\stackrel{\mathrm{y}}{\sim}$, as matrizes $G$ e $R$ são definidas por: $G=A \otimes G_{0}$ e $R=\mid \otimes R_{0}$, onde $\otimes$ denota produto de Kronecker; A é a matriz ( $n \times n)$ de parentesco; I é a matriz identidade ( $n \times n) ; G_{0}$ é a matriz (qxq) de variância e covariância genética aditiva entre as $q$ características, definida por: $G_{0}=\left[\begin{array}{cccc}\sigma_{A_{1}}^{2} & \sigma_{A_{1} A_{2}} & \cdots & \sigma_{A_{1} A_{q}} \\ \sigma_{A_{2} A_{1}} & \sigma_{A_{2}}^{2} & \cdots & \sigma_{A_{2} A_{q}} \\ \vdots & \vdots & \ddots & \\ \sigma_{A_{1} A_{q}} & \sigma_{A_{2} A_{q}} & \cdots & \sigma_{A_{q}}^{2}\end{array}\right]$, em que $\sigma_{A_{i}}^{2}$ é a variância genética aditiva da i-ésima característica e ${ }^{\sigma_{A_{i} A_{j}}}$ é a covariância genética entre as característica i e j, com i $=1,2, \ldots, q$ e $j=1,2, \ldots, q$; $R_{0}$ é a matriz (qxq) de variância e covariância residual entre as q características, 

macho de matrizes de frango de corte: Herdabilidades e correlações. PUBVET, Londrina, V. 5, N. 35, Ed. 182, Art. 1230, 2011.

definida por: $R_{0}=\left[\begin{array}{cccc}\sigma_{\mathrm{e}_{1}}^{2} & \sigma_{\mathrm{e}_{1} \mathrm{e}_{2}} & \cdots & \sigma_{\mathrm{e}_{1} \mathrm{e}_{\mathrm{q}}} \\ \sigma_{\mathrm{e}_{2} \mathrm{e}_{1}} & \sigma_{\mathrm{e}_{2}}^{2} & \cdots & \sigma_{\mathrm{e}_{2} \mathrm{e}_{\mathrm{q}}} \\ \vdots & \vdots & \ddots & \\ \sigma_{\mathrm{e}_{1} \mathrm{e}_{\mathrm{q}}} & \sigma_{\mathrm{e}_{2} \mathrm{e}_{\mathrm{q}}} & \cdots & \sigma_{\mathrm{e}_{\mathrm{q}}}^{2}\end{array}\right]$, em que $\sigma_{\mathrm{e}_{\mathrm{i}}}^{2}$ é a variância residual da iésima característica e ${ }^{\sigma_{\mathrm{e}_{\mathrm{i}} \mathrm{e}_{\mathrm{j}}}}$ é a covariância residual entre as característica i e j, $\operatorname{com} i=1,2, \ldots, q$ e $j=1,2, \ldots, q$.

O vetor de observações $\underset{\sim}{y}$, a matriz de incidência de efeitos fixos $X$, a matriz de incidência de valores genéticos $Z$ e $o$ vetor de valores genéticos ${ }_{\sim}^{u}$ apresentam nq linhas mostrando cada observação individual, ou seja, que cada animal produz q equações. Assim, no modelo animal o número de equações a serem resolvidas equivale ao número de animais vezes o número de características (nq).

O sistema de equações do modelo misto é:

$$
\left[\begin{array}{cc}
X^{\prime} R^{-1} X & X^{\prime} R^{-1} Z \\
Z^{\prime} R^{-1} X & Z^{\prime} R^{-1} Z+G^{-1}
\end{array}\right] \cdot\left[\begin{array}{c}
\hat{\beta} \\
\tilde{u} \\
\sim
\end{array}\right]=\left[\begin{array}{cc}
X^{\prime} R^{-1} y \\
& \sim \\
Z R^{-1} & y \\
& \sim
\end{array}\right]
$$

Nas análises uni-característica e bi-características, foi considerado como efeito fixo (vetor $\underset{\sim}{\beta}$ ) a incubação, e como efeito aleatório (vetor $\underset{\sim}{u}$ ), o valor genético aditivo direto dos animais para todas as características avaliadas.

\section{RESULTADOS E DISCUSSÃO}

A análise descritiva das características analisadas é apresentada na Tab. 1. 
Tabela 1 - Número total de aves (n), Média ( $\bar{X}$ ); Desvio-Padrão (S); Coeficiente de Variação (CV); Menor valor observado (Min) e Maior valor observado (Max).

\begin{tabular}{lllllll}
\hline Característica & $\mathrm{N}$ & $\overline{\mathrm{x}}$ & $\mathrm{S}$ & $\mathrm{CV} \%$ & Min & Max \\
\hline P42 $(\mathrm{g})$ & 5418 & 2545,39 & 236,02 & 9,27 & 1750 & 3300 \\
CPeito $(\mathrm{mm})$ & 3887 & 139,49 & 6,25 & 4,48 & 120 & 157 \\
Larg1 $(\mathrm{mm})$ & 3718 & 84,93 & 6,87 & 8,1 & 63 & 99 \\
Larg2 $(\mathrm{mm})$ & 3893 & 52,85 & 6,87 & 13 & 31 & 74 \\
Fert $(\%)$ & 170 & 74,05 & 26,48 & 35,76 & 0 & 100 \\
Eclo $(\%)$ & 162 & 82,85 & 19,69 & 23,76 & 0 & 100 \\
Nasc $(\%)$ & 169 & 65,19 & 24,25 & 37,2 & 0 & 100 \\
P49 $(\mathrm{g})$ & 2172 & 3345,26 & 267,52 & 7,99 & 2410 & 4114 \\
GP4349 (g) & 2158 & 677,74 & 222,00 & 32,75 & -130 & 1480 \\
CA4349 & 2087 & 2,49 & 0,64 & 25,7 & 1,18 & 5,77
\end{tabular}

P42: peso vivo aos 42 dias de idade; CPeito: comprimento de peito; Larg1: largura maior do peito; Larg2: largura menor do peito; Fert: fertilidade; Eclo: eclodibilidade; Nasc: nascimento; P49: peso vivo aos 49 dias de idade; GP4349: ganho de peso entre os dias 43 e 49; CA4349: conversão alimentar entre os dias 43 e 49.

As correlações entre as características estudadas são apresentadas na Tab. 2. 
Tabela 2 - Número total de aves ( $n)$, correlação fenotípica $\left(r_{P}\right)$, correlação genética $\left(r_{A}\right)$, correlação ambiental $\left(r_{E}\right)$.

\begin{tabular}{lcccc}
\hline & $\mathrm{N}$ & $\mathrm{r}_{\mathrm{P}}$ & $\mathrm{r}_{\mathrm{A}}$ & $\mathrm{r}_{\mathrm{E}}$ \\
\hline P42 x CPeito & 3850 & 0,6149 & 0,6767 & 0,5815 \\
\hline P42 x Larg1 & 3679 & 0,5343 & 0,6365 & 0,4854 \\
\hline P42 x Larg2 & 3839 & 0,4610 & 0,4774 & 0,4336 \\
\hline CPeito x Larg1 & 3702 & 0,4913 & 0,3629 & 0,5932 \\
\hline CPeito x Larg2 & 3860 & 0,3433 & 0,3314 & 0,3664 \\
\hline Larg1 x Larg2 & 3704 & 0,6563 & 0,7820 & 0,6015 \\
\hline P49 x GP4349 & 2158 & 0,79 & 0,4657 & 0,8362 \\
\hline P49 x CA4349 & 2078 & $-0,2640$ & $-0,0776$ & $-0,2898$ \\
\hline GP4349 x CA4349 & 2066 & $-0,3837$ & $-0,9413$ & $-0,3187$ \\
\hline P49 x CPeito & 1777 & 0,2966 & 0,4674 & 0,2512 \\
\hline P49 x Larg1 & 1639 & 0,1632 & 0,3463 & 0,1290 \\
\hline P49 x Larg2 & 1773 & 0,1046 & $-0,1189$ & 0,1388 \\
\hline GP4349 x CPeito & 1771 & 0,0055 & $-0,2917$ & 0,0819 \\
\hline GP4349 x Larg1 & 1634 & $-0,0340$ & $-0,1262$ & $-0,0263$ \\
\hline GP4349 x Larg2 & 1773 & $-0,07$ & $-0,48$ & $-0,03$ \\
\hline CA4349 x CPeito & 1700 & $-0,01$ & 0,13 & $-0,0499$ \\
\hline CA4349 x Larg1 & 1566 & 0,0149 & 0,9999 & $-0,014$ \\
\hline CA4349 x Larg2 & 1695 & 0,0212 & 0,2107 & 0,0031
\end{tabular}

P42: peso vivo aos 42 dias de idade; CPeito: comprimento de peito; Larg1: largura maior do peito; Larg2: largura menor do peito; P49: peso vivo aos 49 dias de idade; GP4349: ganho de peso entre os dias 43 e 49; CA4349: conversão alimentar entre os dias 43 e 49.

De acordo com CARDELLINO \& ROVIRA (1987), o grau de correlação genética entre duas características é importante para controlar diretamente a mudança genética de uma característica quando selecionada por meio de outra.

As características de desempenho tendem a ser fortemente correlacionadas com as características de carcaça segundo CAMPOS \& PEREIRA 

macho de matrizes de frango de corte: Herdabilidades e correlações. PUBVET, Londrina, V. 5, N. 35, Ed. 182, Art. 1230, 2011.

(1999); parece existir forte associação genética entre o peso vivo e as características de carcaça (GAYA, 2006).

As correlações genéticas P42XCPeito, P42XLarg1 e P42XLarg2 foram $0,68,0,64,0,48$, respectivamente, estando aproximadas aos valores obtidos por VAYEGO et al.(2008), 0,68, 0,65 e 0,48. Esses valores indicam que a seleção por peso corporal aos 42 dias de idade influencia positivamente as características de peito, utilizadas para inferir a área de peito da ave (SCHMIDT et al., 2006). Desse modo, aves com peso corporal elevado aos 42 dias de idade tendem a apresentar maior área de peito, o que é interessante para linhagens de corte. (VAYEGO et al. 2008). Entretanto, a seleção para elevado peso corporal deve ser feita criteriosamente, pois aves muito pesadas tendem a reduzir suas características de reprodução. A deposição de gordura abdominal é outra característica que sofre influência indireta da seleção para peso corporal. A seleção para peso vivo pode ser capaz de aumentar os rendimentos de carcaça em frangos de corte (CHAMBERS, 1990). Segundo GAYA et al. (2006), ao selecionar-se para peso vivo, aumenta-se a produção de carne indiretamente.

As correlações fenotípicas P42XCPeito, P42XLarg1 e P42XLarg2 foram 0,$61 ; 0,53 ; 0,39$, respectivamente, correspondendo aos valores $(0,62 ; 0,53$; 0,39 ) obtidos por VAYEGO et al. (2008), sendo consideradas moderadas.

A contribuição das correlações genéticas foi levemente superior à das correlações ambientais (VAYEGO et al.,2008). As correlações genéticas CPeitoXLarg1, CPeitoXLarg2 e Larg1XLarg2, foram medianas a alta e positivas $0,36,0,33,0,78$ estando correspondentes aos valores observados por VAYEGO et al.(2008), 0,43,0,33 e 0,84, respectivamente.

As correlações fenotípicas CPeitoXLarg1 e CPeitoXLarg2 foram moderadas $(0,49 ; 0,34)$, de modo que estão próximas aos valores obtidos por VAYEGO et al.(2008), de 0,52 e 0,34, respectivamente.

A estimativa de correlação genética P49XCA é -0,08, sendo inferior as encontradas na literatura, embora essas apresentem grande variação, com valores entre $-0,23 \pm 0,26$ (LEENSTRA et al., 1986) e 0,56 $\pm 0,74$ (LEENSTRA \& 

macho de matrizes de frango de corte: Herdabilidades e correlações. PUBVET, Londrina, V. 5, N. 35, Ed. 182, Art. 1230, 2011.

PIT, 1988). A estimativa encontrada se aproxima ao resultado de GAYA (2003), que não encontrou importantes associações genéticas entre conversão alimentar e as características de carcaça, que variaram entre -0,14 e 0,10.

A herdabilidade mede o grau de correspondência entre o fenótipo e o valor genético, expressando o grau de confiança do valor fenotípico como indicador do valor genético aditivo (CARDELLINO \& ROVIRA, 1987) portanto, prediz o valor genético aditivo a partir do fenotípico (NUNES, 2007).

As herdabilidades estimadas para as características, através do programa DFREML, são apresentadas na Fig.1.

Figura 1 - Proporção da variância total devida aos efeitos genéticos aditivos diretos (herdabilidade) e aos efeitos residuais

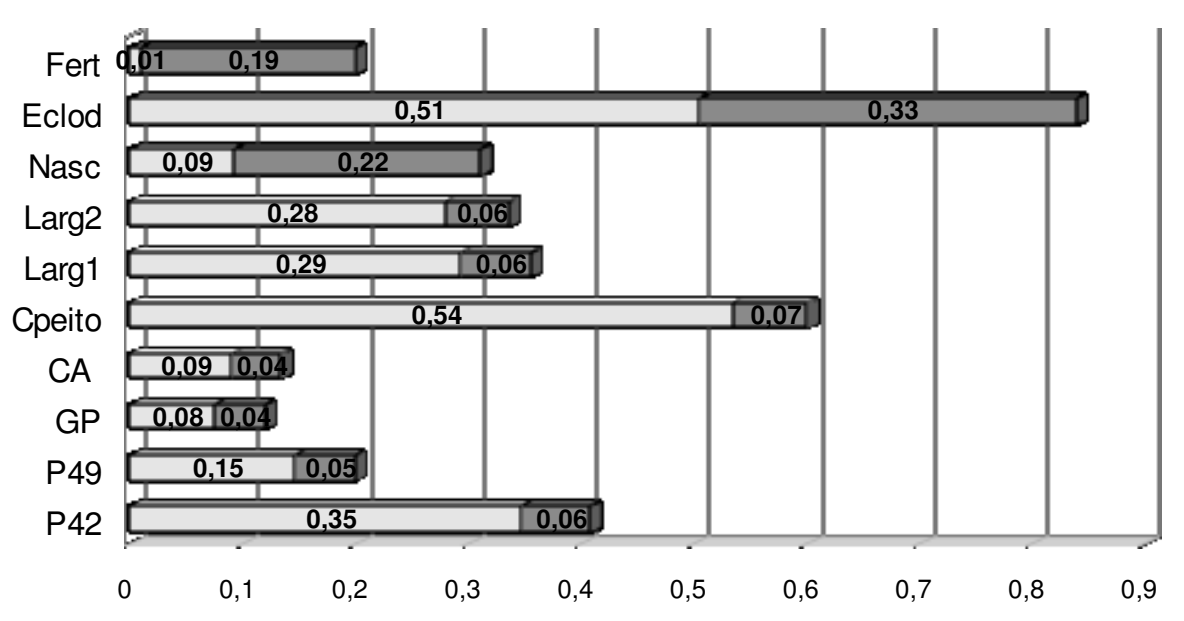

$\square$ Herdabilidade $\square$ Efeitos Residuais

As estimativas de herdabilidade obtidas foram de baixas a moderadas e variaram de 0,01 a 0,53. Segundo CARDELLINO \& ROVIRA (1987), as estimativas para a característica peso corporal podem variar entre 0,30 e 0,50. As estimativas de herdabilidade para peso vivo em diferentes idades variaram

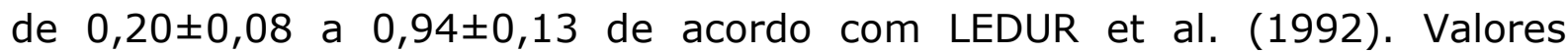
intermediários a estes para a mesma característica foram encontrados por diversos autores (LEENSTRA \& PIT, 1988; ARGENTÃO et al., 2002; RANCE et al., 2002; REZENDE et al., 2005). Valores entre 0,12 e 0,56, foram encontrados para peso corporal aos 28 dias de idade (LEDUR et al., 1992; 

macho de matrizes de frango de corte: Herdabilidades e correlações. PUBVET, Londrina, V. 5, N. 35, Ed. 182, Art. 1230, 2011.

MALLIK et al., 2003 e KUMAR, 2004). Para peso corporal aos 35 dias de idade, estimativas entre 0,28 a 0,63 foram relatadas por LEDUR et al. (1992); KUHLERS \& MCDANIEL (1996); LOPES \& QUAAS (1997) e ARGENTÃO et al. (2002).

As estimativas de herdabilidades obtidas para P42 foi $0,35 \pm 0,06$, ficando dentro da variação $(0,23$ e 0,53) encontrada por LEDUR et al. (1992 e 1994); SCHMIDT et al. (1992); SINGH \& TREHAN (1994); ARGENTÃO et al. (2002); RANCE et al. (2002); KUMAR et al. (2002); AZEVEDO Jr et al. (2002) e MALLIK et al. (2003). BECKER et al. (1984) encontraram valores de herdabilidade diferentes para peso corporal medidos nos machos e nas fêmeas, de 0,61 e 0,88 , respectivamente. A boa capacidade de resposta à seleção do peso vivo é um dos principais fatores responsáveis pela grande evolução alcançada no melhoramento dos frangos de corte (CAMPOS \& PEREIRA, 1999). A estimativa de herdabilidade para P49 foi 0,15 $\pm 0,05$.

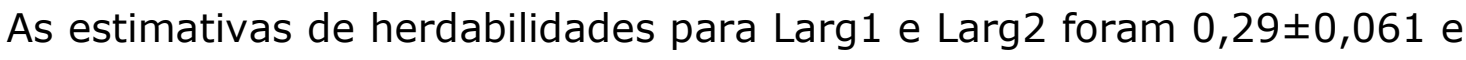
$0,27 \pm 0,057$, respectivamente, estando aproximadas à variação encontrada por

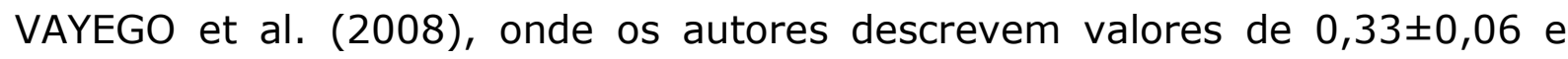
$0,29 \pm 0,05$. Isso indica que essas características podem responder com eficiência à seleção.

A característica CPeito apresentou estimativa de herdabilidade de 0,53 $\pm 0,06$, estando de acordo com o resultado obtido por VAYEGO et al. (2008). Esses resultados são interessantes, pois indicam que ganhos genéticos significativos em quantidade de carne de peito podem ser obtidos, o que é de interesse comercial em se tratando de linhagem de corte.

A estimativa de herdabilidade para CA obtida $(0,09 \pm 0,04)$ está inferior aos valores obtidos no estudo de LEENSTRA \& PIT (1988), onde variaram entre $0,14 \pm 0,18$ e $0,44 \pm 0,15$. Valores intermediários a estes foram encontrados por ARGENTÃO et al. (2002) e GAYA (2003); 0,22 e 0,16 respectivamente. Segundo CAMPOS \& PEREIRA (1999), a conversão alimentar tem apresentado diferentes capacidades de resposta à seleção nos estudos que vêm sendo conduzidos, o que se confirma por meio das estimativas encontradas na 

macho de matrizes de frango de corte: Herdabilidades e correlações. PUBVET, Londrina, V. 5, N. 35, Ed. 182, Art. 1230, 2011.

literatura. A conversão alimentar representa uma característica importante em um programa de seleção, já que os gastos com alimentação representam de 50 a 70\% dos custos nesta atividade (WANG et al., 1991) e desperdícios devem ser evitados (GAYA et al., 2006).

Para GP, a herdabilidade estimada foi de $0,075 \pm 0,04$, sendo inferior ao valor citado por MELO et al. (2004), de 0,16.

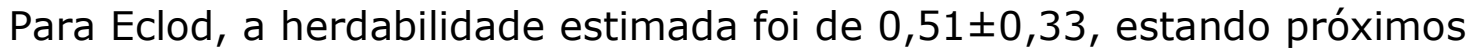
de DAVIS (1955) que obteve uma estimativa de 0,65, sendo superior aos valores citados por outros autores, como CUSTÓDIO (2000), que determinou

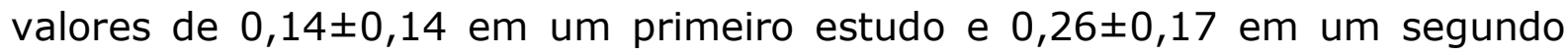

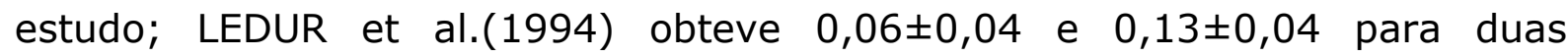
linhagens diferentes; CRITTENDEN \& BOHREN $(1961,1962)$ determinaram herdabilidade de 0,16 para eclodibilidade a partir da variância de galos, em uma linhagem materna.

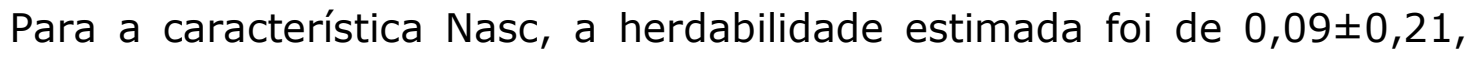
sendo inferior ao obtido por CUSTÓDIO (2000), onde foi obtido valores de $0,18 \pm 0,15$ e $0,26 \pm 0,17$.

A estimativa obtida para Fert foi de $0,01 \pm 0,19$, aproximando-se do encontrado por CRITTENDEN et al. (1957), com o valor de 0,02; sendo inferior para as estimativas obtidas por outros autores: para CUSTÓDIO (1981) o valor

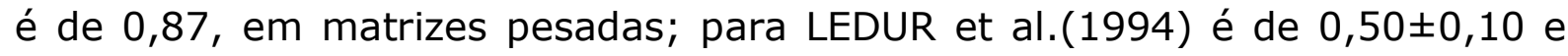
$0,23 \pm 0,05$ para linhagens maternas.

\section{CONCLUSÃO}

Para as estimativas de herdabilidade que foram de moderadas a altas (CPeito, Eclod, P42, L1, L2), indica-se que uma parcela relativamente grande da variação observada nas características é decorrente dos genes de efeito aditivo e que é possível alcançar ganhos genéticos, por meio de seleção, nas aves da linhagem estudada. 
SZINVELSKI, R.G. et al. Parâmetros genéticos para características de produção em linhagem macho de matrizes de frango de corte: Herdabilidades e correlações. PUBVET, Londrina, V. 5, N. 35, Ed. 182, Art. 1230, 2011.

Para as características de correlações genéticas que foram positivas e elevadas, (CAxL1, L1xL2, P42xCPeito, P42xL1，P42xL2, P49xCPeito, P49xGP4349) a seleção feita sobre elas pode ser eficiente.

Tendo em vista a escassez de trabalhos sobre melhoramento genético em frangos de corte para determinadas características, é necessário que se façam mais pesquisas e se invista mais nesse setor, já que a avicultura brasileira tem se destacado ano a ano com números recordes de produção e exportação.

\section{REFERÊNCIAS BIBLIOGRÁFICAS}

ANDRADE, A.N. Avicultura do futuro: uma perspectiva para o século 21. In: REUNIÃO ANUAL DA SOCIEDADE BRASILEIRA DE ZOOTECNIA, 30, 1990, Piracicaba. Anais... Piracicaba: FEALQ, 1990. $p, 71-76$.

ARGENTÃO, C.; MICHELAN FILHO, T.; MARQUES, J. B. et al. Genetic and phenotypic parameters of growth and carcass traits of male line of broilers raised in tropical conditions. In. CONGRESS ON GENETICS APLLIED TO LIVESTOCK PRODUCTION, 7., 2002, Montpellier. Proceedings ...Castanet - Tolosan. Organising comitee WCGALP, 2002.v. 30. p. 333-336.

AZEVEDO P. C., SILVA, M; A., FRIDRICH, A. B. et al. Comparação de metodologias para estimação de parâmetros genéticos em população de aves submetida a seleção. Arquivo Brasileiro de Medicina Veterinaria e Zootecnia, v. 54, p. 518-524, 2002.

BECKER, W A.; SPENCER, J.V.; MIROSH, L.W.; VERSTRATE, J.A. Genetic variation of abdominal fat, body weight, and carcass weight in a female broiler line. Poultry Science, v.63, p.607-611, 1984.

BOARETTO, T. N. Melhoramento genético em frangos de corte. 2009. Revista formação e informação em zootecnia, v.1, n.1, p.11-18.

CAMPOS, E.J; PEREIRA, J.C.C. Melhoramento genético das aves. In: PEREIRA, J.C.C. Melhoramento genético aplicado à produção animal. Belo Horizonte: FEP-MVZ, 1999. Cap.17, p.284-314.

CARDELLINO, R. A., ROVIRA, J. Mejoramento genético animal. Montevideo. Hemisfério Sur, 1987. 253p.

CHAMBERS, J.R. Advantages and disadvantages of genetic improvement of meat-type poultry and possible solutions. In: SIMPÓSIO TÉCNICO DE MATRIZES DE CORTE, 1., 1995, Chapecó. Anais... Chapecó: Associação Catarinense de Avicultura, 1995. p, 120- 131.

CRITTENDEN, L.B.; BOHREN, B.B. The effects of current egg production, time in production, age of pullet, and inbreeding on hatchability and hatching time. Poultry Science, Champaign, v.41, p.426-433, 1962. 
CRITTENDEN, L.B.; BOHREN, B.B. The genetic and environmental effects of hatching time, egg weight and holding time or hatchability. Poultry Science, Champaign, v.40, p.1736-1750, 1961.

CRITTENDEN, L.B.; BOHREN, B.B.; ANDERSON, V.L. Genetic variance and covariance of the components of hatchability in New Hampshires. Poultry Science, v. 36, n.1; p.90-103, 1957.

CUSTÓDIO, R. W. S. Estimativas de repetibilidades e herdabilidades para produção de ovos e pintos com acasalamentos hierárquicos em galinhas para corte. Brasília, 2000. Pesquisa Agropecuária Brasileira, v.35, n.5, p.967-974.

CUSTÓDIO, R. W. S. Obtenção de repetibilidade na produção de ovos e pintos de um dia em galinhas para corte. Brasília, 2000. Pesquisa Agropecuária Brasileira, vol.35, n.5.

DAVIS, G.T. Influence of oxygen concentration on hatchability and on selecting for hatchability. Poultry Science, v.34, p.107-113, 1955.

FIGUEIREDO, E. A. P.; SCHMIDT, G. S.; ROSA, P. S.; LEDUR, M. C. O programa de melhoramento genético de aves da EMBRAPA. Belo Horizonte, 2000. In. SIMPÓSIO NACIONAL DE MELHORAMENTO ANIMAL, 3. Anais... Belo Horizonte. UFMG, 2000. p. 58 - 62.

GAYA, L. G.; MOURÃO, G. B.; FERRAZ, J. B. S. Aspectos genético-quantitativos de características de desempenho, carcaça e composição corporal em frangos. Santa Maria, 2006. Ciência Rural, Santa Maria, v.36, n.2, p.709-716.

GAYA, L. G. e FERRAZ, J. B. S. Aspectos genético-quantitativos da qualidade da carne em frangos. Santa Maria, 2006. Ciência Rural, v.36, n.1, p.349-356.

GAYA, L.G. Estudo genético da deposição de gordura abdominal e de características de desempenho, carcaça e composição corporal em linhagem macho de frangos de corte. 2003. 99f. Dissertação (Mestrado em Zootecnia) - Curso de Pós Graduação em Zootecnia, Faculdade de Zootecnia e Engenharia de Alimentos da Universidade de São Paulo. Capturado em 28 de jul. 2010. Online. Disponível na Internet http://www.teses.usp.br/teses/disponiveis/74/74131/tde-12042004-164232/.

KUHLERS, D. L.; MCDANIEL, G. R. Estimates of herdabilities and genetic correlations between tibial dyschondroplasia expression and body weight at two ages in broilers. Poultry Science, $v$ 75, p. 959-961, 1996.

KUMAR, S.; SINGH, R. P.; SINGH, S. K. et al. Study of genetic parameters of broiler sire line. Indian Journal of Poultry Science, v. 37, p. 221-225, 2002.

KUMAR, V. P. Heritability and genetic correlation estimates in broiler chicken. Indian Journal of Poultry Science, v. 39, p. 264-265, 2004.

LEDUR, M.C.; SCHMIDT, G. S.; FIGUEIREDO, E. A. P.; et al. Parâmetros genéticos e fenotípicos em linhagens de aves selecionadas para corte. Brasília, 1994. Pesquisa Agropecuária Brasileira, v.29, n. 3, p.503-508.

LEDUR, M. C.; SCHMIDT, G. S.; AVILA, V. S. et al. Parâmetros genéticos e fenotípicos para peso corporal em diferentes idades em linhagens de frango de corte. Revista Brasileira de Zootecnia, v. 21, p. 667-673, 1992. 
LEENSTRA, F.R. et al. Fat deposition in a broiler sire strain I.phenotypic and genetic variation in, and correlations between abdominal fat, body weight and feed conversion. Poultry Science, Savoy, v.65, p.1225-1235, 1986.

LEENSTRA, F.R.; PIT, R. Fat deposition in a broiler sire line: heritability of and genetic correlations among body weight, abdominal fat, and feed conversion. Poultry Science, Savoy, v.67, p.1-9, 1988.

LOPES, P. S.; QUAAS, R. L. Estimação de parâmetros genéticos em aves de corte. Revista Brasileira de Zootecnia, v. 26, p. 1119-1123, 1997.

MALLIK, B. K.; MISHRA, P. K.; MISHRA, S. C. et al. Evaluation of genetic parameters in a synthetic broiler chicken flock due to selection for high six week body weight. Indian Journal of Poultry Science, v. 38, p. 225-229, 2003.

MELO, C. M. R.; NONES, K.; LEDUR, M. C.; et al. Parâmetros genéticos e fenotípicos de caracteres de desempenho e carcaça em um cruzamento experimental de linhagens de aves de corte e de postura. Campo Grande, MS; 2004. 41a Reunião Anual da Sociedade Brasileira de Zootecnia, p.1-4.

NUNES, B. N. Parâmetros genéticos e fenotípicos de peso corporal ao abate e características de carcaça em cruzamentos recíprocos de aves. Dissertação (Mestre em Genética e Melhoramento Animal) - Faculdade de Ciências Agrárias e Veterinárias, Campus de Jaboticabal - UNESP, 2007.

PEREIRA, F. A. Programa de Melhoramento genético de aves e suínos na Agroceres. 1996 p.116-122.

RANCE, K.A. et al. Genetic and phenotypic relationships between and within support and demand tissues in a single line of broiler chicken. British Poultry Science, Roslin, v.43, p.518$527,2002$.

REZENDE et al. Estimativas de parâmetros genéticos e fenotípicos de características de desempenho e carcaça em uma linhagem macho de frangos. In: CONFERÊNCIA APINCO DE CIÊNCIA E TECNOLOGIA AVÍCOLAS, 2005, Santos. Anais...Santos: FACTA, 2005. p.160.

SCHMIDT, G. S.; FIGUEIREDO, E. A. P. Situação atual e perspectivas do programa de melhoramento genético de aves do CNPSA/EMBRAPA. Concórdia, SC; 1996. I Simpósio Brasileiro de Melhoramento Animal, p. 99-104.

SINGH, R.; TREHAN, P. K. Genetic and phenotypic parameters of body and muscle weights and abdominal fat in meat-type chicken. Indian Journal of Animal Science, v. 64, p. 388-392, 1994.

VAYEGo, S. A.; DiOnello, N. J. L.; FIGUeiRedo, E. A. P. Estimativas de parâmetros e tendências genéticas para algumas características de importância econômica em linhagem paterna de frangos de corte sob seleção. 2008. Revista Brasileira de Zootecnia, v.37, n.7, p.1230-1235.

VAYEGO, S. A. Uso de modelos mistos na avaliação genética de linhagens de matrizes de frangos de corte. Curitiba, 2007. Dissertação (Doutor em Genética) - Universidade Federal do Paraná.

WANG, L. et al. Genetic correlations among growth, feed and carcass traits of broiler sire and dam populations. Poultry Science, Savoy, v.70, p.719-725, 1991. 